\title{
Size-Dependent Optical Properties of Colloidal PbS Quantum Dots
}

\author{
Iwan Moreels, ${ }^{\dagger, *}$ Karel Lambert, ${ }^{\dagger}$ Dries Smeets, ${ }^{\neq, \pi}$ David De Muynck, ${ }^{\S}$ Tom Nollet, ${ }^{\dagger}$ José C. Martins, ${ }^{\perp}$ \\ Frank Vanhaecke, ${ }^{\S}$ André Vantomme, ${ }^{\neq}$Christophe Delerue, Guy Allan," and Zeger Hens ${ }^{\dagger, *}$ \\ ${ }^{\dagger}$ Physics and Chemistry of Nanostructures, Ghent University, Krijgslaan 281-S12, B-9000 Ghent, Belgium, ${ }^{\ddagger}$ Institute for Nuclear and Radiation Physics and INPAC, \\ Celestijnenlaan 200D, K.U. Leuven, B-3001 Leuven, Belgium, ${ }^{\S}$ Laboratory of Analytical Chemistry, Ghent University, Krijgslaan 281-S12, B-9000 Ghent, Belgium, ${ }^{\perp}$ NMR \\ and Structure Analysis Unit, Ghent University, Krijgslaan 281-S4bis, B-9000 Ghent, Belgium, and "Institut d'Electronique, de Microélectronique et de Nanotechnologie, \\ F-59652 Villeneuve d'Ascq Cedex, France. "Current address: Université de Montréal.
}

$\mathrm{F}$ or more than 25 years, interest in colloidal semiconductor nanocrystals or quantum dots (Qdots) has kept growing at an increasing pace, mostly driven by the exceptional optical properties these Qdots possess. Several materials can now be produced by well-controlled colloidal syntheses, ${ }^{1}$ and their basic material properties $^{2,3}$ (most importantly the particle size and its influence on the band gap) have been thoroughly investigated. Consequently, more and more applicationoriented research is being pursued, primarily in the direction of Qdot-based biolabeling, ${ }^{4,5}$ LEDs, ${ }^{6}$ lasers, ${ }^{7,8}$ and solar cells. ${ }^{9}$ It is expected that these applications will benefit greatly from the enhanced and tunable Qdot optical properties.

In order to fabricate and optimize these devices, it is highly desirable that one has access to the concentration $c_{0}$ of the suspended Qdots. Through Beer's law, $c_{0}$ can be conveniently determined from the absorbance spectrum of a suspension if the Qdot molar extinction coefficient $\varepsilon$ is known. At present, $\varepsilon$ values for cadmium chalcogenide, ${ }^{10-12} \operatorname{InP},{ }^{13} \operatorname{InAs},,^{14}$ and lead chalcogenide ${ }^{15-17}$ Qdots have been reported. Unfortunately, the data also show that the methods used to determine $\varepsilon$ still vary strongly among research groups, resulting for instance in different empirical size dependencies of $\varepsilon$ at the band gap for the same material. ${ }^{10,12,16,17}$

Experimental methods to determine $\varepsilon$ and $c_{0}$ are separable into two main groups. Often, the value of $\varepsilon$ at the band gap is used, ${ }^{12,15,17}$ either at a single wavelength or integrated over the first absorption peak. On the other hand, several groups have recently shown that the value of $\varepsilon$ at energies far above the band gap bears more practi-

ABSTRACT We quantitatively investigate the size-dependent optical properties of colloidal PbS nanocrystals or quantum dots (Qdots), by combining the Qdot absorbance spectra with detailed elemental analysis of the Qdot suspensions. At high energies, the molar extinction coefficient $\varepsilon$ increases with the Qdot volume $d^{3}$ and agrees with theoretical calculations using the Maxwell-Garnett effective medium theory and bulk values for the Qdot dielectric function. This demonstrates that quantum confinement has no influence on $\varepsilon$ in this spectral range, and it provides an accurate method to calculate the Qdot concentration. Around the band gap, $\varepsilon$ only increases with $d^{1 \cdot 3}$, and values are comparable to the $\varepsilon$ of $\mathrm{PbSe} Q \mathrm{Qdots}$. The data are related to the oscillator strength $f_{\text {if }}$ of the band gap transition and results agree well with theoretical tight-binding calculations, predicting a linear dependence of $f_{\text {if }}$ on $d$. For both PbS and PbSe Qdots, the exciton lifetime $\tau$ is calculated from $f_{\text {if }}$. We find values ranging between 1 and $3 \mu \mathrm{s}$, in agreement with experimental literature data from time-resolved luminescence spectroscopy. Our results provide a thorough general framework to calculate and understand the optical properties of suspended colloidal quantum dots. Most importantly, it highlights the significance of the local field factor in these systems.

KEYWORDS: semiconductor nanocrystals $\cdot$ lead chalcogenide $\cdot \mathrm{PbSe} \cdot \mathrm{molar}$ extinction coefficient · oscillator strength · exciton lifetime

cal significance. More specifically, for CdSe, ${ }^{11}$ InAs, ${ }^{14}$ and $\mathrm{PbSe}^{16}$ Qdots, bulk-like optical properties were measured at 350 , 450 , and $400 \mathrm{~nm}$, respectively. These were modeled by the Maxwell-Garnett (MG) effective medium theory, ${ }^{18,19}$ applicable to dispersed particles in a (transparent) dielectric host. Using the dielectric function of the respective bulk semiconductor and the solvent refractive index, the MG model yielded an $\varepsilon$, or equivalently an absorption coefficient $\mu$ or cross section $C_{a b s}$, which agrees well with the experimental data at short wavelengths. This demonstrates that quantum confinement has no influence on $\varepsilon$ in this spectral range. Hence, $\varepsilon$ is not affected by the particle size dispersion, improving the accuracy of the reported data.

The successful application of the MG effective medium theory also highlights that it can give us a better quantitative insight in the Qdot optical properties and their
*Address correspondence to
iwan.moreels@ugent.be,
zeger.hens@ugent.be.
Received for review July 24, 2009
and accepted September 8, 2009.
Published online September 25,
2009.
10.1021/nn900863a CCC: $\mathbf{1 0 . 7 5}$
@ 2009 American Chemical Society
3023-3030 - 2009 

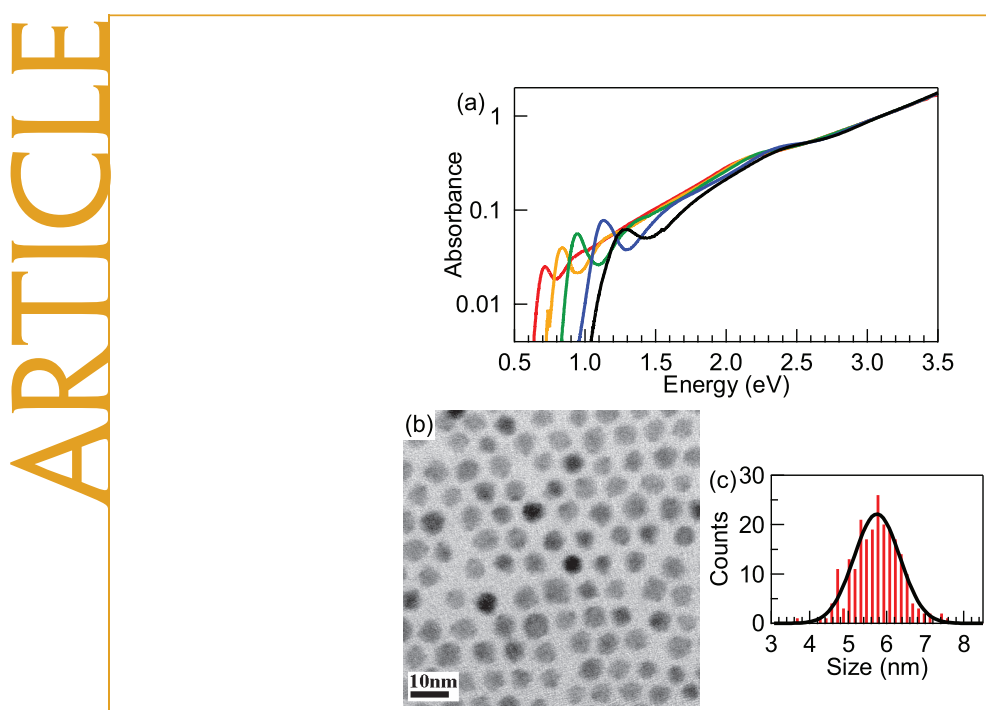

Figure 1. (a) Series of absorbance spectra of PbS Qdots suspended in $\mathrm{C}_{2} \mathrm{Cl}_{4}$, normalized at $3.1 \mathrm{eV}$. All spectra coincide at high energies. (b) Overview TEM image of a typical PbS Qdot suspension. (c) Corresponding size histogram. The mean size equals $5.7 \mathrm{~nm}$, with a size dispersion of $10 \%$.

evolution with the particle size $d .^{20}$ Indeed, for PbSe Qdots, we recently reported that, in contrast with the bulk-like $\mu$ at high energies, the energy-integrated $\mu$ at the band gap shows a strong size dependence, resulting in an oscillator strength $f_{\text {if }}$ per particle, which increases approximately linearly with $d .{ }^{16}$ Experimental data are in agreement with theoretical tight-binding calculations, ${ }^{21}$ and they for instance demonstrate that, for Qdots in the strong confinement regime, a constant $f_{\text {if }}$ should not be expected. ${ }^{22,23}$

In this paper, we apply the methods derived previously ${ }^{16}$ to calculate the optical properties of colloidal $\mathrm{PbS}$ Qdots. First, the PbS Qdot concentration $c_{0}$ is carefully determined by elemental analysis of the $\mathrm{Pb}$ concentration of digested PbS Qdot suspensions, in combination with a measurement of the atomic $\mathrm{Pb} / \mathrm{S}$ ratio. Beer's law then yields the PbS Qdot $\varepsilon$, which is analyzed both at high energies and at energies around the band gap. We make a detailed comparison with the $\varepsilon$ of PbSe Qdots. Practically, this allows one to determine which material, from an optical perspective, might be more suitable for further applications. Theoretically, it improves the insight in the underlying physics determining the optical properties, and it confirms the applicability of the MG effective medium theory. Finally, we expand the current analysis methods to obtain the exciton lifetime $\tau$ for both materials and compare these calculated values to experimental literature data.

\section{RESULTS}

Determination of the Sizing Curve. $\mathrm{PbS}$ is a narrow gap semiconductor, with a bulk band gap of $0.41 \mathrm{eV}$, and a large exciton Bohr radius (18 $\mathrm{nm}$ ). Consequently, the $\mathrm{PbS}$ Qdot band gap $E_{0}$ can be tuned over the entire NIR spectral range. Figure 1a shows a series of absorbance spectra for PbS Qdots, normalized at $3.1 \mathrm{eV}(400 \mathrm{~nm})$. It demonstrates that the synthesis allows the prepara-

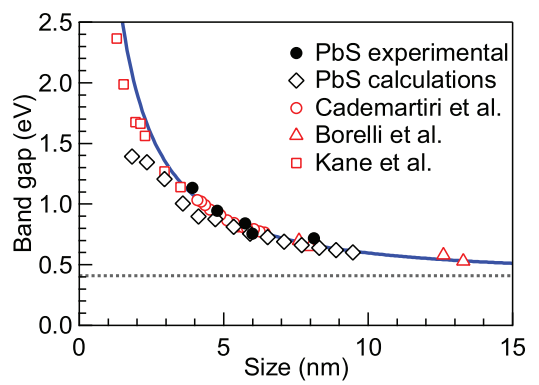

Figure 2. Relation between the PbS Qdot band gap and the particle size. The experimental data (O) agree well with literature values $(\bigcirc, \triangle)$ and tight-binding calculations (our data $\diamond$, literature data $\square$ ). To the set of experimental data, a sizing curve is fitted (full line). The dotted line denotes the bulk $\mathrm{PbS}$ band gap (0.41 eV).

tion of particles with $E_{0}$ varying between 0.71 and 1.28 eV (1740-970 nm). A typical transmission electron microscopy (TEM) image (Figure 1b) illustrates that the PbS Qdots have a spherical shape. An analysis of the size (Figure 1c) yields $d=5.7 \mathrm{~nm}$, with a relative size dispersion $\sigma_{\mathrm{d}}=10 \%$ for the particle suspension shown in Figure $1 \mathrm{~b}$.

The value of $d$ is measured for five samples and correlated with $E_{0}$ to construct a sizing curve. Figure 2 shows that the data correspond well with those of Cademartiri et al. ${ }^{15}$ and Borrelli and Smith. ${ }^{24} \mathrm{~A}$ fit to both our data and the experimental literature values yields the following result (size range fitted: $d$ $=3.9-13.3 \mathrm{~nm}$ ):

$$
E_{0}=0.41+\frac{1}{0.0252 d^{2}+0.283 d}
$$

The construction of this sizing curve allows us to determine $d$ directly from $E_{0}$, avoiding a lengthy TEM analysis for each sample synthesized. Experimental results agree well with theoretical tight-binding calculations (Figure 2). Both predict an $E_{0}$ scaling mainly with $d^{-1}$. This is similar to the size dependence of $E_{0}$ for PbSe Qdots, ${ }^{16,21}$ albeit that PbS Qdots are typically larger than PbSe Qdots for a given $E_{0}$. Note that, although we have not synthesized particles with a size below $3.5 \mathrm{~nm}$, the extrapolation of the sizing curve still agrees well with calculated data of Kane et al. ${ }^{25}$

Assessment of the Sample Purity. When determining the Qdot concentration using elemental analysis of the cation and anion, it is crucial that samples are free of unreacted precursors. As described by Cademartiri et al., ${ }^{15}$ lead chloride $\left(\mathrm{PbCl}_{2}\right)$ is insoluble in apolar solvents, and trace amounts still present after synthesis and purification precipitate slowly from the suspension. In accordance with their procedure, we use powder X-ray diffraction (XRD) to demonstrate the absence of $\mathrm{PbCl}_{2}$. A typical X-ray diffractogram (XRD) is shown in Figure 3a. It confirms that all $\mathrm{PbCl}_{2}$ is removed, as its presence would be revealed by, for instance, reflections at 22.9, 35.8 , and $46.5^{\circ} 2 \theta .^{26}$ The position of the reflections aris- 

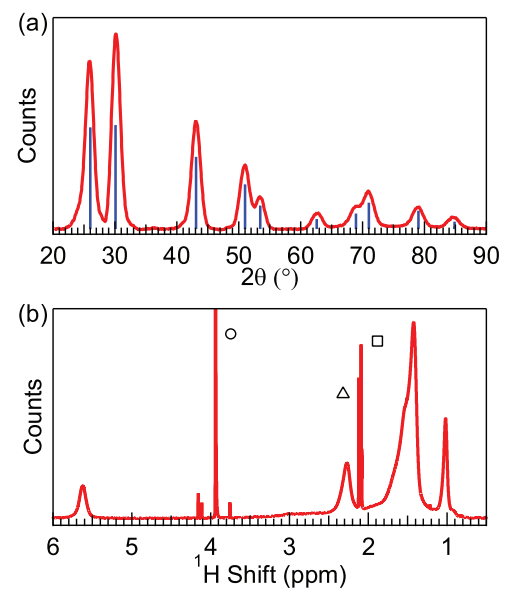

Figure 3. (a) X-ray diffractogram of PbS Qdots. The PbS Qdot crystal structure corresponds to the bulk $\mathrm{PbS}$ rocksalt structure (vertical lines). In addition, no reflections due to unreacted $\mathrm{PbCl}_{2}$ are observed. (b) ${ }^{1} \mathrm{H}$ NMR spectrum of a $\mathrm{PbS}$ Qdot suspension in toluene- $d_{8}$. Apart from $\mathrm{CH}_{2} \mathrm{Br}_{2}(\bigcirc)$, residual toluene $(\square)$ and possible methanol traces $(\triangle)$, no sharp resonances arising from unreacted OLA-S and/or TOPS are observed. The broad resonances correspond to the OA ligands.

ing from the PbS Qdots agrees well with the expected values for bulk PbS (rocksalt structure, lattice constant $a$ $=5.936 \AA){ }^{26}$ demonstrating that these colloidal Qdots maintain their crystal structure when the size is decreased from bulk down to the nanoscale.

To confirm that the suspensions are free of sulfurbased precursors (oleylamine-sulfur complexes, OLA-S, and/or tri- $n$-octylphosphine sulfide, TOPS), PbS Qdots are suspended in deuterated toluene (toluene$d_{8}$ ) and a quantitative proton nuclear magnetic resonance ( ${ }^{1} \mathrm{H}$ NMR) spectrum is measured. ${ }^{16,27}$ Figure $3 \mathrm{~b}$ shows that, apart from a sharp singlet due to dibromomethane $\left(\mathrm{CH}_{2} \mathrm{Br}_{2}, 3.94 \mathrm{ppm}, \mathrm{O}\right)$, a quintuplet due to residual toluene (2.09 ppm, $\square$ ) and a singlet possibly due to methanol (2.12 ppm, $\triangle$ ), no sharp signals arising from OLA-S and/or TOPS are observed. The broad resonances in fact arise from oleic acid (OA) ligands bound to the PbS Qdot surface. A similar ${ }^{1} \mathrm{H}$ NMR spectrum is observed for OA-capped PbSe Qdots. ${ }^{27}$

Both the XRD and NMR results clearly demonstrate that our samples are free of unreacted precursors. This enables us to reliably determine PbS Qdot concentrations using elemental analysis.

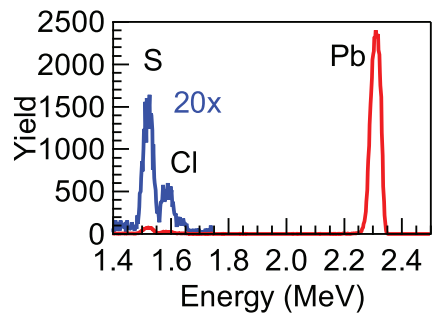

Figure 4. RBS spectrum of a typical PbS Qdot close-packed thin film. The three peaks can be attributed to $\mathrm{He}^{+}$ions that have collided with $\mathrm{Pb}, \mathrm{S}$, and $\mathrm{Cl}$. The $\mathrm{S}$ and $\mathrm{Cl}$ peaks have been multiplied by 20 for clarity.
TABLE 1. $\mathrm{Pb} / \mathrm{S}, \mathrm{Cl} / \mathrm{Pb}$, and $\mathrm{Cl} / \mathrm{Pb}_{\mathrm{e}}$ Ratio Obtained for the Five Samples Used for RBS ( $\mathrm{Pb}_{\mathrm{e}}$ Denotes the Excess $\mathrm{Pb}$ Atoms)

\begin{tabular}{cccc}
$\boldsymbol{d}(\mathbf{n m})$ & $\mathbf{P b} / \mathbf{S}$ & $\mathbf{C l} / \mathbf{P b}$ & $\mathbf{C l} / \mathbf{P b}_{\mathbf{e}}$ \\
\hline 3.7 & 1.37 & 0.49 & 1.80 \\
4.1 & 1.23 & 0.38 & 2.07 \\
4.9 & 1.26 & 0.37 & 1.83 \\
5.9 & 1.27 & 0.32 & 1.48 \\
6.8 & 1.28 & 0.34 & 1.55 \\
\hline
\end{tabular}

Qdot Composition and Concentration. We use Rutherford backscattering spectrometry (RBS) ${ }^{28,29}$ to measure the $\mathrm{Pb} / \mathrm{S}$ atomic ratio $R$ of five samples. Figure 4 shows a typical RBS spectrum, measured on $5.9 \mathrm{~nm}$ PbS Qdots. Three peaks are observed, corresponding to $\mathrm{He}^{+}$ions, which have collided with $\mathrm{Pb}, \mathrm{S}$, and $\mathrm{Cl}$. From the area under the peaks, we determine the respective atomic ratios. Table 1 summarizes the results. We estimate the uncertainty on the data to be $5 \%$. The sample with the smallest size shows a $\mathrm{Pb} / \mathrm{S}$ ratio of 1.37:1. For all other samples, we obtain, within experimental error, a constant ratio of 1.26:1. These results demonstrate that, like PbSe Qdots, ${ }^{16}$ PbS Qdots are nonstoichiometric, showing a systematic $\mathrm{Pb}$ excess $\mathrm{Pb}_{\mathrm{e}}$. Interestingly, a significant amount of $\mathrm{Cl}$ is also present despite the absence of unreacted $\mathrm{PbCl}_{2}$, at ratios which are consistent with results of Cademartiri et al. ${ }^{30}$ From the approximate 2:1 ratio of $\mathrm{Cl}$ to $\mathrm{Pb}_{\mathrm{e}}$, we conclude that the excess $\mathrm{Pb}$ atoms most probably reside at the Qdot surface, with the $\mathrm{Cl}$ atoms bound to them.

Assuming a spherical particle, the number of atoms per Qdot $N$ equals

$$
N=\frac{4 \pi}{3}\left(\frac{d}{a}\right)^{3}
$$

Taking the $\mathrm{Pb} / \mathrm{S}$ stoichiometry $R$ into account, the $\mathrm{Pb}$ weight concentration $C_{\mathrm{Pb}}(\mathrm{mg} / \mathrm{L})$, determined with inductively coupled plasma mass spectrometry (ICP-MS), and the $\mathrm{Pb}$ molar mass $M_{\mathrm{Pb}}=207.2 \mathrm{~g} / \mathrm{mol}$ then yield the Qdot concentration $c_{0}(\mu \mathrm{mol} / \mathrm{L})$ :

$$
c_{0}=\frac{10^{3}}{N} \frac{1+R}{R} \frac{C_{\mathrm{Pb}}}{M_{\mathrm{Pb}}}
$$

Table 2 shows the results for the four samples measured. Note that, in accordance with the RBS measurements, we take $R=1.37: 1$ for the $3.6 \mathrm{~nm}$ PbS Qdots,

TABLE 2. Weight Concentration $C_{\mathrm{Pb}}$, Obtained with ICP$\mathrm{MS}, \mathrm{Pb} / \mathrm{S}$ Ratio $R$ (In Accordance with the RBS Data), Resulting Qdot Concentration $c_{0}$, and Absorbance of the Suspension at $400 \mathrm{~nm} A_{400}$

\begin{tabular}{cccccc}
$\boldsymbol{d}(\mathbf{n m})$ & $\boldsymbol{\sigma}_{\mathrm{d}}(\%)$ & $\boldsymbol{C}_{\mathrm{pb}}(\mathbf{m g} / \mathbf{L})$ & $\boldsymbol{R}$ & $\boldsymbol{C}_{0}(\boldsymbol{\mu M})$ & $A_{400}\left(\mathrm{~cm}^{-1}\right)$ \\
\hline 3.6 & 9.6 & 5.78 & 1.37 & 0.054 & 0.563 \\
4.5 & 10.2 & 7.08 & 1.26 & 0.033 & 0.707 \\
5.4 & 8.8 & 7.65 & 1.26 & 0.021 & 0.761 \\
6.5 & 10.7 & 4.98 & 1.26 & 0.008 & 0.504 \\
\hline
\end{tabular}



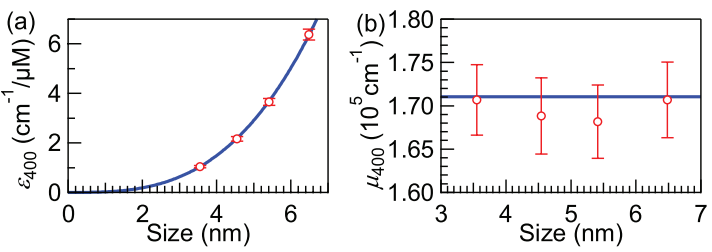

Figure 5. (a) At $400 \mathrm{~nm}$, the PbS Qdot molar extinction coefficient $\varepsilon_{400}$ increases with $d^{3}$. (b) Consequently, the absorption coefficient $\mu_{400}$ is size-independent. Results are in agreement with a theoretical $\mu$, calculated using the bulk $\mathrm{PbS}$ dielectric function (full line).

while for the other samples, $R=1.26: 1$ is used. $\sigma_{\mathrm{d}}$ is listed as well to highlight that the size-dependent optical properties are calculated starting from samples with a small size dispersion.

$\mathrm{PbS}$ Qdot Molar Extinction Coefficient at $400 \mathrm{~nm}$. From the absorbance of an equal amount of PbS Qdots, the molar extinction coefficient is derived using Beer's law. The absorbance at $400 \mathrm{~nm} A_{400}$ is listed in Table 2. At this wavelength, the molar extinction coefficient scales with the nanocrystal volume (Figure 5a):

$$
\varepsilon_{400}=(0.0233 \pm 0.0001) d^{3} \mathrm{~cm}^{-1} / \mu \mathrm{M}
$$

with $d$ in nanometers. The following relation relates the experimental $\varepsilon_{400}$ to the PbS Qdot absorption coefficient $\mu_{400}$ ( $N_{\mathrm{A}}$ : Avogadro's constant):

$$
\mu_{400}=\frac{6}{\pi d^{3}} \frac{\ln (10) \varepsilon_{400}}{N_{\mathrm{A}}}
$$

Figure $5 \mathrm{~b}$ shows the resulting size-independent $\mu_{400}$ for PbS Qdots. The uncertainty on the data is estimated to be $3 \%$.

Using the MG effective medium theory, $\mu$ can also be calculated at a given wavelength $\lambda$ from the dielectric function of the semiconductor $\left(\varepsilon_{\mathrm{R}}+i \varepsilon_{1}\right)$ and the solvent $\left(\varepsilon_{\mathrm{s}}=n_{\mathrm{s}}^{2}\right)$ :

$$
\begin{gathered}
\mu=\frac{2 \pi}{n_{\mathrm{s}} \lambda}\left|f_{\mathrm{LF}}\right|^{2} \varepsilon_{\mathrm{l}} \\
f_{\mathrm{LF}}=\frac{3 \varepsilon_{\mathrm{s}}}{\varepsilon_{\mathrm{R}}+i \varepsilon_{\mathrm{l}}+2 \varepsilon_{\mathrm{s}}}
\end{gathered}
$$

Here, $f_{\mathrm{LF}}$ denotes the (complex) local field factor. Equation 6 shows that it can strongly modify the absorption of a material when it is dispersed as small particles in a host with a different dielectric function (this is a consequence of dielectric confinement). ${ }^{31,32}$ Using bulk values for the $\mathrm{PbS}$ dielectric function at $400 \mathrm{~nm}\left(\varepsilon_{R}=\right.$ $\left.4.53, \varepsilon_{1}=26.4\right)^{33}$ and the tetrachloroethylene $\left(\mathrm{C}_{2} \mathrm{Cl}_{4}\right)$ refractive index $\left(n_{s}=1.53\right),{ }^{34}$ eq 6 yields a sizeindependent $\mu_{400}=1.71 \times 10^{5} \mathrm{~cm}^{-1}$ (Figure 5b, full line). Using the theoretical $\mu_{400}$, we find the following theoretical expression for the molar extinction coefficient: $\varepsilon_{400}=0.0234 d^{3} \mathrm{~cm}^{-1} / \mu \mathrm{M}$. Clearly, both $\mu_{400}$ and $\varepsilon_{400}$ are in excellent agreement with the experimental data.
As for PbSe Qdots, ${ }^{16}$ the cubic size dependence of $\varepsilon_{400}$ demonstrates that the PbS Qdot optical properties at energies well above the band gap are not influenced by quantum confinement. This was already apparent from the normalized absorbance spectra, as all spectra coincide at high energies (Figure 1). These results have an important practical consequence. As they are independent of size and therefore size dispersion, $\varepsilon_{400}$ allows for a very accurate determination of the Qdot concentration $c_{0}$. This method is therefore to be preferred over a procedure based on the Qdot absorbance at the band gap. ${ }^{12,17}$

PbS Qdot Optical Properties at the Band Gap. We calculate the molar extinction at the band gap $\varepsilon_{\text {gap }}$ by integrating the first absorption peak on an energy scale. ${ }^{16}$ The four ICP-MS samples directly yield $\varepsilon_{\text {gap }}$ from an integration of the $\varepsilon$ spectrum. However, we have measured the absorbance spectrum of 10 additional PbS Qdot samples suspended in $\mathrm{C}_{2} \mathrm{Cl}_{4}$, and for these samples, $\varepsilon_{\text {gap }}$ can be determined using $\varepsilon_{400}\left(A_{\text {gap }}\right.$ is the area of the energy-integrated first absorption peak):

$$
\varepsilon_{\text {gap }}=\frac{A_{\text {gap }}}{A_{400}} \varepsilon_{400}
$$

The results are shown in Figure 6a. Note that the data are more scattered than the values at $400 \mathrm{~nm}$, confirming that a determination of $c_{0}$ using Beer's law and $\varepsilon$ at $400 \mathrm{~nm}$ will be more accurate.

In contrast with $\varepsilon_{\text {gap }}$ which depends on $n_{\mathrm{s}}$ through $f_{\mathrm{LF}}$, the Qdot oscillator strength $f_{\text {if }}$ is a material property. It therefore provides a better insight into the intrinsic Qdot optical properties. Approximating the Qdot as
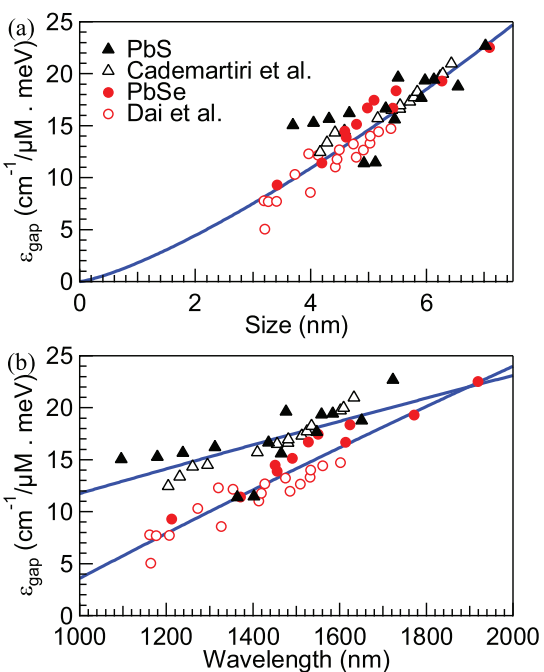

Figure 6. (a) At the band gap, we observe a similar molar extinction coefficient $\varepsilon_{\text {gap }}$ for PbS and PbSe Qdots of equal size. Both scale with $d^{1.3}$. The results are in agreement with $\mathrm{PbS}$ data of Cademartiri et al. (after correction for their observed $\mathrm{Pb} / \mathrm{S}$ Qdot ratio) and $\mathrm{PbSe}$ data of Dai et al. (b) When comparing $\mathrm{PbS}$ and $\mathrm{PbSe}$ Qdots of equal band gap, $\mathrm{PbS}$ Qdots clearly have a larger $\varepsilon_{\text {gap }}$. This is due to their different sizing curve, resulting in larger $\mathrm{PbS}$ Qdots for a given band gap. 
a two-level system around the band gap, it can be calculated directly from $\varepsilon_{\text {gap }}\left(m_{\mathrm{e}}\right.$ : free electron mass, $\varepsilon_{0}$ : electric constant): ${ }^{16}$

$$
f_{\text {if }}=\frac{2 \varepsilon_{0} c m_{\mathrm{e}} n_{\mathrm{s}}}{\pi N_{\mathrm{A}} \hbar e} \frac{1}{\left|f_{\mathrm{LF}}\right|^{2}} \ln (10) \varepsilon_{\text {gap }}
$$

As PbS Qdots are suspended in $\mathrm{C}_{2} \mathrm{Cl}_{4}$, we use $n_{\mathrm{s}}=$ $1.49,{ }^{34}$ valid at NIR wavelengths. In accordance with our recently reported calculations for PbSe Qdots, we estimate $\left|f_{\mathrm{LF}}\right|^{2}$ using bulk data for $\mathrm{PbS}$, valid around the PbS band gap: $\varepsilon_{\mathrm{R}}=20.25 \gg \varepsilon_{\mid \cdot}{ }^{33}$ This yields $\left|f_{\mathrm{LF}}\right|^{2}=$ 0.0725 . Figure 7 shows the resulting $f_{\text {if }}$ as a function of d. An approximately linear dependence is observed, and results agree well with theoretical tight-binding calculations.

\section{DISCUSSION}

Comparison of $\varepsilon_{\text {gap }}$ with PbSe Qdots and Literature Data. Figure 6a compares the PbS Qdot data to $\varepsilon_{\text {gap }}$ of Cademartiri et al. ${ }^{15}$ We have slightly adjusted their values to account for two differences. First, although they observed that PbS Qdots are nonstoichiometric in an earlier report, ${ }^{30}$ it appears that this was not considered in their more recent calculation of $\varepsilon_{\text {gap }}$ (data were based solely on a $\mathrm{Pb}$ elemental analysis ${ }^{15}$ ). Taking their reported $\mathrm{Pb} / \mathrm{S}$ ratio of $1.3-1.5$, it is estimated that their $\varepsilon_{\text {gap }}$ is $c a .15-20 \%$ too small. We therefore choose to increase their values by $17.5 \%$. Second, they report a wavelength-integrated value $\varepsilon_{\text {gap }, \lambda}$. This can be converted to an energyintegrated $\varepsilon_{\text {gap }}:{ }^{16}$

$$
\varepsilon_{\text {gap }}=\frac{e E_{0}^{2}}{h c} \varepsilon_{\text {gap }, \lambda}
$$

After conversion, we see that both data sets agree well, confirming the validity of our method to calculate $\varepsilon_{\text {gap }}$.

For further comparison, we suspended $11 \mathrm{PbSe}$ Qdot samples in $\mathrm{C}_{2} \mathrm{Cl}_{4}$ and calculated $\varepsilon_{\text {gap }}$ from eq 8 using $\varepsilon_{400}=0.0311 d^{3} \mathrm{~cm}^{-1} / \mu \mathrm{M}\left(\mu_{400}=2.27 \times 10^{5} \mathrm{~cm}^{-1}\right)$. These equations are obtained using the MG effective medium theory and bulk PbSe data at $400 \mathrm{~nm}$

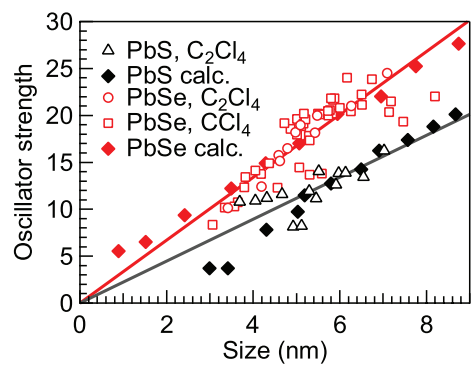

Figure 7. The experimental $\mathrm{PbS}$ and $\mathrm{PbSe} \mathrm{Qdot}$ oscillator strength increases approximately linearly with size. $\mathrm{PbSe}$ Qdots suspended in $\mathrm{CCl}_{4}(\square)$ yield the same values as $\mathrm{PbSe}$ Qdots suspended in $\mathrm{C}_{2} \mathrm{Cl}_{4}(\bigcirc)$. Data for $\mathrm{PbS}$ are, however, $33 \%$ smaller $(\triangle)$. For both materials, experimental values agree with with tight-binding calculations $(\diamond)$.
$\left(\varepsilon_{\mathrm{R}}=-10.67, \varepsilon_{1}=20.54\right){ }^{35}$ as optical properties are also bulk-like at this wavelength. In addition to our data, Dai et al. ${ }^{17}$ recently reported a wavelength-integrated $\varepsilon_{\text {gap }, \lambda}$ (normalized by a standard peak width of $K=60$ $\mathrm{nm})$. Again, this is readily converted to an energy integrated $\varepsilon_{\text {gap }}$ through eq 10 . After conversion, both data sets agree well. Interestingly, Figure 6a shows that PbS and PbSe Qdots of equal size have a comparable $\varepsilon_{\text {gap }}$. A power law fit to both the PbS and PbSe Qdot data yields

$$
\varepsilon_{\text {gap }}=1.8 d^{1.3} \mathrm{~cm}^{-1} \mathrm{meV} / \mu \mathrm{M}
$$

From a practical perspective, $\varepsilon_{\text {gap }}$ at a given wavelength is often more relevant. In Figure $6 b$, we therefore compare $\mathrm{PbS}$ and $\mathrm{PbSe}$ Qdots of equal $E_{0}$. It shows that, due to the different size dependence of $E_{0}, \mathrm{PbS}$ Qdots, which are larger for a given $E_{0}$, also have the larger $\varepsilon_{\text {gap }}$. This implies that, for optical applications using colloidal lead chalcogenide Qdots, PbS might be a more suitable material than $\mathrm{PbSe}$.

Comparison of $f_{\text {if }}$ with the PbSe Qdot $f_{\text {if }}$. For PbSe Qdots suspended in $\mathrm{C}_{2} \mathrm{Cl}_{4}$, we estimate $\left|f_{\mathrm{LF}}\right|^{2}$ at the band gap using $\varepsilon_{\mathrm{R}}=27.04{ }^{35}$ yielding $\left|f_{\mathrm{LF}}\right|^{2}=0.0446$. This value is $38 \%$ smaller than $f_{\mathrm{LF}}$ for PbS Qdots, due to the larger $\varepsilon_{\mathrm{R}}$ of PbSe. Figure 7 compares $f_{\text {if }}$ for PbS and PbSe Qdots. First, for PbSe Qdots, we indeed observe that samples suspended in $\mathrm{CCl}_{4}$ (data taken from our previous report ${ }^{16}$ ) and in $\mathrm{C}_{2} \mathrm{Cl}_{4}$ show the same $f_{\text {if, }}$ confirming that $f_{\text {if }}$ is a solvent-independent material property. Second, comparing $\mathrm{PbSe}$ to $\mathrm{PbS}$ values, we observe that $f_{\text {if }}$ is significantly smaller (ca. 33\%) for PbS Qdots (note that this decreases in $f_{\text {if }}$ is, however, almost exactly compensated by the difference in $f_{\mathrm{LF}}$; this is why a similar $\varepsilon_{\text {gap }}$ is still observed in Figure 6a). Both data sets agree well with the tight-binding calculations, and we observe that the PbS and PbSe oscillator strength scales approximately linearly with $d$.

$$
\begin{gathered}
f_{\text {if,PbS }}=(2.23 \pm 0.09) d \\
f_{\text {if,PbSe }}=(3.35 \pm 0.05) d
\end{gathered}
$$

It shows that a size-independent $f_{\text {if }}$ should not be expected for Qdots in the strong confinement regime, as predicted by earlier calculations within an effective mass approximation. ${ }^{22,23}$

Calculation of the Exciton Lifetime. The oscillator strength is related to the exciton decay rate $\tau^{-1}$ by Fermi's golden rule $\left(\omega=E_{0} / \hbar\right.$ is the band gap transition frequency): ${ }^{36}$

$$
\tau^{-1}=\frac{e^{2}}{2 \pi \varepsilon_{0} c^{3} m_{\mathrm{e}}} n_{\mathrm{s}}\left|f_{\mathrm{LF}}\right|^{2} \omega^{2} \frac{f_{\text {if }}}{g}
$$

Here, $g$ denotes the degeneracy of the band gap exciton ( $g=64$ for lead chalcogenide Qdots). We hereby assume that the oscillator strength of spontaneous emission is equal to the oscillator strength of the band gap absorption $f_{\text {if }}$, reduced by $g$.

VOL. $3 \cdot$ NO. $10 \cdot 3023-3030 \cdot 2009$ 
Using eq 9, we can determine $\tau^{-1}$ directly from $\varepsilon_{\text {gap }}$ hereby avoiding the need to calculate $f_{\mathrm{LF}}$ :

$$
\tau^{-1}=\frac{e}{\pi^{2} N_{A} \hbar c^{2}} n_{s}^{2} \omega^{2} \frac{\ln (10) \varepsilon_{\text {gap }}}{g}
$$

Figure 8a shows that, with the exception of the smallest PbSe Qdots ( $d<5 \mathrm{~nm}$ ), $\tau^{-1}$ increases linearly with $\omega$. This linear trend is in agreement with experimental data obtained on CdSe and CdTe Qdots and, from a theoretical viewpoint, is explained by a sizeindependent dipole matrix element: ${ }^{37}$

$$
|<f| \mathbf{p}|i>|^{2}=\frac{3 \hbar m_{\mathrm{e}} \omega}{2} f_{\text {if }}
$$

The deviation from linearity in the case of small PbSe Qdots therefore indicates that $|<f| \mathbf{p}|i>|^{2}$ becomes size-dependent for $d<5 \mathrm{~nm}$.

The exciton lifetime $\tau$ is shown in Figure $8 \mathrm{~b}$ as a function of $d$. For PbS Qdots suspended in $\mathrm{C}_{2} \mathrm{Cl}_{4}, \tau$ varies linearly between 1 and $1.8 \mu$ s over the entire range of sizes that we have analyzed. For PbSe Qdots in $\mathrm{CCl}_{4}, \tau$ varies between 2.0 and $3.4 \mu \mathrm{s}$, while somewhat smaller values are obtained when they are suspended in $\mathrm{C}_{2} \mathrm{Cl}_{4}$, due to a larger local field factor in $\mathrm{C}_{2} \mathrm{Cl}_{4}$. Interestingly, the PbSe Qdot $\tau$ shows a minimal value for a diameter of about $5 \mathrm{~nm}$. These data are in reasonable agreement with experimental values obtained through time-resolved luminescence measurements for both $\mathrm{PbS}^{38}$ and PbSe Qdots. ${ }^{39,40}$ More specifically, the decreasing trend in $\tau$ for small PbSe Qdots has also been recently observed by Kigel et al., ${ }^{39}$ who measured radiative lifetimes between 4.97 and 2.74 $\mu$ s for PbSe Qdots of $d=2.7-4.7 \mathrm{~nm}$,
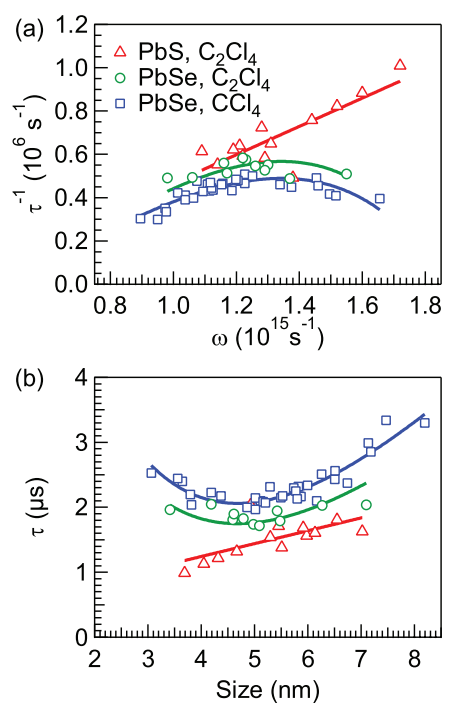

Figure 8. (a) For both $\mathrm{PbS}(\triangle)$ and $\mathrm{PbSe}$ Qdots suspended in $\mathrm{C}_{2} \mathrm{Cl}_{4}(\bigcirc)$ and $\mathrm{CCl}_{4}(\square)$, the decay rate $\tau^{-1}$ increases linearly with the band gap frequency $\omega$ (except for the smallest PbSe Qdots, $d<5 \mathrm{~nm}$ ). (b) The resulting lifetime $\tau$ for PbSe Qdots varies between 1.7 and $3.4 \mu \mathrm{s}$. Values for PbS Qdots are somewhat smaller $(1-1.8 \mu \mathrm{s})$. and Oron et al., ${ }^{40}$ who find $2.5-0.9 \mu$ s for the fast component of their luminescence decay $(d=$ $3.2-4.3 \mathrm{~nm})$.

\section{CONCLUSIONS}

PbS Qdots are synthesized according to literature methods. Their size is determined with TEM and related to the Qdot band gap to construct a sizing curve. With RBS, we show that the Qdots are nonstoichiometric, with an excess of $\mathrm{Pb}$ atoms most probably located at the Qdot surface. A layer of $\mathrm{Cl}$ atoms is bound to these excess $\mathrm{Pb}$ atoms. Combination of the $\mathrm{Pb} / \mathrm{S}$ ratio with elemental ICP-MS analysis of the atomic $\mathrm{Pb}$ concentration enables us to calculate the Qdot concentration. Through Beer's law, we then obtain the Qdot molar extinction coefficient $\varepsilon$.

At energies far above the band gap, $\varepsilon$ scales with the Qdot volume. Its value is compared to a theoretical calculation using the MG effective medium theory and the bulk semiconductor dielectric function. Both show excellent agreement. This has a large practical advantage: Qdot concentrations can be accurately determined from $\varepsilon$ in this spectral range, irrespective of size dispersion.

Around the band gap, the energy-integrated $\varepsilon_{\text {gap }}$ scales with $d^{1.3}$. The PbS Qdot $\varepsilon_{\text {gap }}$ is clearly larger than $\varepsilon_{\text {gap }}$ for PbSe Qdots of equal band gap, rendering PbS Qdots more suitable for optical applications due to their stronger absorption at the band gap. The Qdot oscillator strength at the band gap $f_{\text {if }}$ can be calculated directly from $\varepsilon_{\text {gap }}$. We observe that the PbS Qdot $f_{\text {if }}$ is $33 \%$ smaller than $f_{\text {if }}$ for PbSe Qdots, in agreement with tightbinding calculations. Both scale approximately linearly with size.

From $f_{\text {if }}$, or equivalently $\varepsilon_{\text {gap }}$, the exciton lifetime $\tau$ is determined. We find values of $1.7-3.4 \mu \mathrm{s}$ for PbSe Qdots and 1-1.8 $\mu$ s for PbS Qdots, in reasonable agreement with recent literature data. Interestingly, in contrast with PbS Qdots, small PbSe Qdots show a decrease in lifetime with increasing size, a trend which has recently also been observed using time-resolved photoluminescence spectroscopy. Although experimental values still differ by a factor of $2-3$, they clearly show a trend which is in line with our results. In this respect, these calculated lifetimes provide a useful reference in the interpretation of experimental data.

In conclusion, we present a thorough general framework to understand and quantify the optical properties of colloidal semiconductor Qdots, both at high energies and at the band gap. Most importantly, the correspondence with the MG effective medium theory highlights the importance of the local field factor and the effects of dielectric confinement in the determination of $\varepsilon, f_{\mathrm{if}}$ and $\tau$. 


\section{METHODS}

Materials. $\mathrm{C}_{2} \mathrm{Cl}_{4}$ ( $\left.\geq 99 \%\right), \mathrm{CH}_{2} \mathrm{Br}_{2}$ (99+\%), and OA (90\%) were purchased from Sigma-Aldrich. TOP (97\%) was purchased from STREM Chemicals and OLA (80-90\%) from Acros. Sulfur (99.999\%, S) and $\mathrm{PbCl}_{2}$ (99.999\%) were purchased from AlfaAesar. Methanol $(\mathrm{MeOH})$, butanol $(\mathrm{BuOH})$, and toluene were all of quality "for synthesis"; concentrated (65\%) nitric acid $\left(\mathrm{HNO}_{3}\right)$ was of quality "normatom ultrapure". These products were ordered from VWR. Toluene- $d_{8}(99.96 \%$ deuterated) was purchased from CortecNet.

Colloidal PbS Qdot Synthesis. The PbS Qdot synthesis is based on the procedure described by Cademartiri et al. ${ }^{30}$ The entire synthesis is carried out under a nitrogen atmosphere to avoid oxidation. First, a stock solution of $0.16 \mathrm{~g}$ of $\mathrm{S}$, dissolved in $15 \mathrm{~mL}$ of OLA, is heated to $120^{\circ} \mathrm{C}$ for $30 \mathrm{~min}$ and afterward cooled to room temperature. In a three-neck flask, $0.56 \mathrm{~g}$ of $\mathrm{PbCl}_{2}$ is mixed with $10 \mathrm{~mL}$ of OLA and heated to $75-150{ }^{\circ} \mathrm{C}$ (depending on the desired particle size). After $30 \mathrm{~min}, 3 \mathrm{~mL}$ of the $S$ stock solution is mixed with $3 \mathrm{~mL}$ of OLA and injected into the three-neck flask. To increase the available size range, $225 \mu \mathrm{L}$ of TOP may be added to the synthesis. When the desired growth time is reached (typically $1-20 \mathrm{~min}$ ), the reaction is quenched by addition of $20 \mathrm{~mL}$ of $\mathrm{BuOH}$ and $10 \mathrm{~mL}$ of $\mathrm{MeOH}$. After centrifugation and decantation, the PbS Qdots are suspended in toluene.

After synthesis, the PbS Qdot organic ligand shell, consisting of OLA, is substituted by OA, by adding $400 \mu \mathrm{L}$ of OA to the Qdot suspension, followed by precipitation with an excess of $\mathrm{MeOH}$ and centrifugation. After decantation, the Qdots are resuspended in toluene and precipitated again with $\mathrm{MeOH}$ to further remove impurities. After this second centrifugation and decantation step, the OA-capped Qdots are finally suspended in toluene and stored under nitrogen and in the dark.

$\mathrm{PbSe}$ Qdots are synthesized based on the procedure developed by Murray et al. ${ }^{41}$ The synthesis is described in detail elsewhere. $^{16}$

Determination of the PbS Qdot Size. We determine the mean diameter $d$ and relative standard deviation $\sigma_{d}$ (also called size disper sion) of five PbS Qdot suspensions with TEM. The samples are prepared by dipping a TEM grid in a diluted Qdot suspension. We use multiple images to measure the area of 200-400 particles, from which the equivalent circular diameter is calculated. Values of $d$ and $\sigma_{d}$ are calculated directly from the individual sizes but agree well with the results of a Gaussian fit to the histogram.

Purity of the PbS Qdot Suspensions. In accordance with the procedure of Cademartiri et al., ${ }^{15}$ samples are stored for several months to ensure that all traces of $\mathrm{PbCl}_{2}$ (which is insoluble in apolar solvents) have precipitated. Hereafter, decantation separates the $\mathrm{PbCl}_{2}$ from the suspension containing the Qdots, and a powder $\mathrm{X}$-ray diffractogram is measured to determine whether $\mathrm{PbCl}_{2}$ is still present.

In addition, ${ }^{1} \mathrm{H}$ NMR is used to detect possible traces of OLA-S and/or TOPS complexes. Samples are prepared by drying a toluene suspension of PbS Qdots, followed by resuspension in deuterated toluene (toluene- $d_{8}$ ). ${ }^{1} \mathrm{H}$ NMR spectra are measured at 295 K, using a Bruker DRX 500 spectrometer equipped with a TBI Z-gradient probe head. Absolute concentrations of all organic species in the suspensions are determined by adding a known amount ( $2 \mu \mathrm{L}$ ) of $\mathrm{CH}_{2} \mathrm{Br}_{2}$ to the suspension and collecting the spectra under quantitative conditions. ${ }^{16,27}$

Determination of the PbS Qdot Composition and Concentration. We calculate the concentration of PbS Qdots $c_{0}$ from the elemental concentrations of $\mathrm{Pb}$ and $\mathrm{S}$, in combination with a calculation of the number of atoms $N$ per Qdot of given $d$. First, a known amount of four PbS Qdot samples is digested in $\mathrm{HNO}_{3}$. The $\mathrm{Pb}$ concentration is then determined with ICP-MS. The measurements are performed with a PerkinElmer SCIEX Elan 5000 inductively coupled plasma mass spectrometer.

We are not able to accurately determine the S concentration with ICP-MS for several reasons. First, sulfur displays a high first ionization energy, resulting in a low sensitivity of Ar-based ICP-MS for this element. Second, signals arising from $\mathrm{O}_{2}^{+}$and $\mathrm{HO}_{2}^{+}$ molecular ions are isobaric with signals arising from $\mathrm{S}$ ions and further hamper an accurate determination. Third, the preliminary digestion of $\mathrm{PbS}$ Qdot samples using concentrated $\mathrm{HNO}_{3}$ im- plies the risk of losing sulfur from the solution due to the formation of volatile $\mathrm{H}_{2} \mathrm{~S}$. Therefore, we use $\mathrm{RBS}^{28,29}$ to measure the $\mathrm{Pb} / \mathrm{S}$ atomic ratio $R$ of five independent samples. As PbS Qdots are synthesized from $\mathrm{PbCl}_{2}$, the $\mathrm{Cl} / \mathrm{Pb}$ ratio is determined, as well. RBS samples are prepared by dropcasting a small amount of PbS Qdots on a MgO substrate, hereby forming a 100-200 nm close-packed thin film. The measurements are performed with a 2.5 $\mathrm{MeV} \mathrm{He}^{+}$ion beam and two solid-state detectors placed at a backscattering angle of 165 and $167.6^{\circ}$, respectively. The high energy of the beam ensures that $\mathrm{S}$ and $\mathrm{Cl}$, atoms with a comparable mass, provide resolved signals. From the backscattered yield, $R$ is determined:

$$
R=\frac{A_{\mathrm{Pb}}}{A_{\mathrm{S}}}\left(\frac{Z_{\mathrm{S}}}{Z_{\mathrm{Pb}}}\right)^{2}
$$

$A_{\mathrm{Pb}}$ denotes the area of the peak resulting from backscattering at $\mathrm{Pb}$ atoms, $A_{\mathrm{S}}$ equals the area of the $\mathrm{S}$ peak, $Z_{\mathrm{Pb}}$ and $Z_{\mathrm{S}}$ are the atomic number of $\mathrm{Pb}$ and $\mathrm{S}$, respectively. A similar expression yields the $\mathrm{Cl} / \mathrm{Pb}$ ratio. As both $\mathrm{RBS}$ detectors yield similar results, we report the average value.

Absorbance Measurements. To determine the relation between the Qdot TEM size $d$ and the band gap $E_{0}$, small amounts of the $\mathrm{PbS}$ Qdots used for the TEM measurements are dried and resuspended in $1 \mathrm{~mL}$ of $\mathrm{C}_{2} \mathrm{Cl}_{4}$. The absorbance spectra are measured with a Perkin-Elmer Lambda 950 UV-vis-NIR spectrophotometer. We use a black walled self-masking microcell with a path length of $I=1 \mathrm{~cm}$. $E_{0}$ is determined from the spectral position of the first absorption peak.

For the determination of $\varepsilon$, identical amounts of PbS Qdots as used for the ICP-MS measurements are dried and resuspended in $1 \mathrm{~mL}$ of $\mathrm{C}_{2} \mathrm{Cl}_{4}$. From the absorbance $A$ and $c_{0}$, obtained from ICP-MS and RBS data, $\varepsilon$ is determined using Beer's law: $A=\varepsilon c_{0}$ l.

Acknowledgment. This project is funded by the IWTVlaanderen (SBO-Metacel), the Belgian Science Policy Office (IAP P6/10), the EU Seventh Framework Program (EU-FP7 ITN Herodot), and the FWO-Vlaanderen (G.0.144.08.N.10). Olivier Janssens (Department of Solid State Sciences, Ghent University) is acknowledged for measuring the XRD spectra.

\section{REFERENCES AND NOTES}

1. de Mello Donega, C.; Liljeroth, P.; Vanmaekelbergh, D. Physicochemical Evaluation of the Hot-Injection Method, a Synthesis Route for Monodisperse Nanocrystals. Small 2005, 1, 1152-1162.

2. Efros, A. L.; Rosen, M. The Electronic Structure of Semiconductor Nanocrystals. Annu. Rev. Mater. Sci. 2000, 30, 475-521.

3. Sargent, E. H. Infrared Quantum Dots. Adv. Mater. 2005, 17 515-522.

4. Michalet, X.; Pinaud, F. F.; Bentolila, L. A.; Tsay, J. M.; Doose, S.; Li, J. J.; Sundaresan, G.; Wu, A. M.; Gambhir, S. S.; Weiss, S. Quantum Dots for Live Cells, In Vivo Imaging, and Diagnostics. Science 2005, 307, 538-544.

5. Gill, R.; Zayats, M.; Willner, I. Semiconductor Quantum Dots for Bioanalysis. Angew. Chem., Int. Ed. 2008, 47, 7602-7625.

6. Rogach, A. L.; Gaponik, N.; Lupton, J. M.; Bertoni, C.; Gallardo, D. E.; Dunn, S.; Pira, N. L.; Paderi, M.; Repetto, P.; Romanov, S. G.; et al. Light-Emitting Diodes with Semiconductor Nanocrystals. Angew. Chem., Int. Ed. 2008, 47, 6538-6549.

7. Eisler, H. J.; Sundar, V. C.; Bawendi, M. G.; Walsh, M.; Smith, H. I.; Klimov, V. Color- Selective Semiconductor Nanocrystal Laser. Appl. Phys. Lett. 2002, 80, 4614-4616.

8. Hoogland, S.; Sukhovatkin, V.; Howard, I.; Cauchi, S.; Levina, L.; Sargent, E. H. A Solution-Processed $1.53 \mu \mathrm{M}$ Quantum Dot Laser with Temperature-Invariant Emission Wavelength. Opt. Express 2006, 14, 3273-3281.

9. Sargent, E. H. Infrared Photovoltaics Made by Solution Processing. Nat. Photonics 2009, 3, 325-331.

10. Rajh, T.; Micic, O. I.; Nozik, A. J. Synthesis and Characterization of Surface-Modified Colloidal CdTe Quantum Dots. J. Phys. Chem. 1993, 97, 11999-12003. 
11. Leatherdale, C. A.; Woo, W. K.; Mikulec, F. V.; Bawendi, M. G. On the Absorption Cross Section of CdSe Nanocrystal Quantum Dots. J. Phys. Chem. B 2002, 106, 7619-7622.

12. Yu, W. W.; Qu, L. H.; Guo, W. Z.; Peng, X. G. Experimental Determination of the Extinction Coefficient of CdTe, CdSe, and CdS Nanocrystals. Chem. Mater. 2003, 15, 2854-2860.

13. Talapin, D. V.; Gaponik, N.; Borchert, H.; Rogach, A. L.; Haase, M.; Weller, H. Etching of Colloidal InP Nanocrystals with Fluorides: Photochemical Nature of the Process Resulting in High Photoluminescence Efficiency. J. Phys. Chem. B 2002, 106, 12659-12663.

14. Yu, P. R.; Beard, M. C.; Ellingson, R. J.; Ferrere, S.; Curtis, C.; Drexler, J.; Luiszer, F.; Nozik, A. J. Absorption Cross-Section and Related Optical Properties of Colloidal InAs Quantum Dots. J. Phys. Chem. B 2005, 109, 7084-7087.

15. Cademartiri, L.; Montanari, E.; Calestani, G.; Migliori, A.; Guagliardi, A.; Ozin, G. A. Size-Dependent Extinction Coefficients of PbS Quantum Dots. J. Am. Chem. Soc. 2006, 128, 10337-10346.

16. Moreels, I.; Lambert, K.; De Muynck, D.; Vanhaecke, F.; Poelman, D.; Martins, J. C.; Allan, G.; Hens, Z. Composition and Size-Dependent Extinction Coefficient of Colloidal PbSe Quantum Dots. Chem. Mater. 2007, 19, 6101-6106.

17. Dai, Q.; Wang, Y.; Li, X.; Zhang, Y.; Pellegrino, D. J.; Zhao, M.; Zou, B.; Seo, J.; Wang, Y.; Yu, W. W. Size-Dependent Composition and Molar Extinction Coefficient of PbSe Semiconductor Nanocrystals. ACS Nano 2009, 3, 1518-1524.

18. Maxwell Garnett, J. C. Colours in Metal Glasses and in Metallic Films. Philos. Trans. R. Soc. London, Ser. A 1904, 203, 385-420.

19. Sihvola, A. Two Main Avenues Leading to the Maxwell Garnett Mixing Rule. J. Electromagn. Wave 2001, 15, 715725.

20. Moreels, I.; Hens, Z. On the Interpretation of Colloidal Quantum-Dot Absorption Spectra. Small 2008, 4, 1866-1868.

21. Allan, G.; Delerue, C. Confinement Effects in PbSe Quantum Wells and Nanocrystals. Phys. Rev. B 2004, 70, 245321.

22. Kayanuma, Y. Quantum-Size Effects of Interacting Electrons and Holes in Semiconductor Microcrystals with Spherical Shape. Phys. Rev. B 1988, 38, 9797-9805.

23. Wang, Y.; Herron, N. Nanometer-Sized Semiconductor Clusters-Materials Synthesis, Quantum Size Effects, and Photophysical Properties. J. Phys. Chem. 1991, 95, 525-532.

24. Borrelli, N. F.; Smith, D. W. Quantum Confinement of PbS Microcrystals in Glass. J. Non-Cryst. Solids 1994, 180, 25-31.

25. Kane, R. S.; Cohen, R. E.; Silbey, R. Theoretical Study of the Electronic Structure of PbS Nanoclusters. J. Phys. Chem. 1996, 100, 7928-7932.

26. Wyckoff, R. W. G. Crystal Structures, 2nd ed.; Interscience Publishers: New York, 1963.

27. Moreels, I.; Fritzinger, B.; Martins, J. C.; Hens, Z. Surface Chemistry of Colloidal PbSe Nanocrystals. J. Am. Chem. Soc. 2008, 130, 15081-15086.

28. Chu, W.; Mayer, J.; Nicolet, M. Backscattering Spectrometry; Academic Press: New York, 1978.

29. Taylor, J.; Kippeny, T.; Rosenthal, S. J. Surface Stoichiometry of CdSe Nanocrystals Determined by Rutherford Backscattering Spectroscopy. J. Clust. Sci. 2001, $12,571-582$

30. Cademartiri, L.; Bertolotti, J.; Sapienza, R.; Wiersma, D. S.; von Freymann, G.; Ozin, G. A. Multigram Scale, Solventless, and Diffusion-Controlled Route to Highly Monodisperse PbS Nanocrystals. J. Phys. Chem. B 2006, 110, 671-673.

31. Takagahara, T. Effects of Dielectric Confinement and Electron-Hole Exchange Interaction on Excitonic States in Semiconductor Quantum Dots. Phys. Rev. B 1993, 47, 4569-4585.

32. Ricard, D.; Ghanassi, M.; Schanneklein, M. C. Dielectric Confinement and the Linear and Nonlinear-Optical Properties of Semiconductor-Doped Glasses. Opt. Commun. 1994, 108, 311-318.
33. Kanazawa, H.; Adachi, S. Optical Properties of PbS. J. Appl. Phys. 1998, 83, 5997-6001.

34. Marvin, H. H. The Selective Transmission and the Dispersion of the Liquid Chlorides. Phys. Rev. 1912, 34, 161-186.

35. Suzuki, N.; Sawai, K.; Adachi, S. Optical Properties of PbSe. J. Appl. Phys. 1995, 77, 1249-1255.

36. Merzbacher, E. Quantum Mechanics, 2nd ed; Wiley International Edition: New York, 1970

37. van Driel, A. F.; Allan, G.; Delerue, C.; Lodahl, P.; Vos, W. L.; Vanmaekelbergh, D. Frequency-Dependent Spontaneous Emission Rate from CdSe and CdTe Nanocrystals: Influence of Dark States. Phys. Rev. Lett. 2005, 95, 236804.

38. Warner, J. H.; Thomsen, E.; Watt, A. R.; Heckenberg, N. R.; Rubinutein-Dunlop, H. Time- Resolved Photoluminescence Spectroscopy of Ligand-Capped PbS Nanocrystals. Nanotechnology 2005, 16, 175-179.

39. Kigel, A.; Brumer, M.; Maikov, G.; Sashchiuk, A.; Lifshitz, E. The Ground-State Exciton Lifetime of PbSe Nanocrystal Quantum Dots. Superlattices Microstruct. 2009, 46, 272-276.

40. Oron, D.; Aharoni, A.; de Mello Donega, C.; van Rijssel, J.; Meijerink, A.; Banin, U. Universal Role of Discrete Acoustic Phonons in the Low-Temperature Optical Emission of Colloidal Quantum Dots. Phys. Rev. Lett. 2009, 102, 177402.

41. Murray, C. B.; Sun, S. H.; Gaschler, W.; Doyle, H.; Betley, T. A.; Kagan, C. R. Colloidal Synthesis of Nanocrystals and Nanocrystal Superlattices. IBM J. Res. Dev. 2001, 45, 47-56. 